\title{
Estudios bíblicos del NT: \\ Panorama histórico e implicancias para la teología sistemática
}

\author{
Fernando Ramos \\ FACULTAD DE TEOLOGÍA \\ PONTIFICIA UNIVERSIDAD CATÓLICA DE CHILE
}

\section{Premisa}

El estudio de la Sagrada Escritura es una tarea que está permanentemente actualizándose y a su vez está abriendo nuevos horizontes y desafíos. Las características del Nuevo Testamento aportan ulteriores desafíos que contribuyen a la complejidad de este tipo de estudios. Por una parte, aunque en menor medida que el Antiguo Testamento, la pluralidad de autores plantea la pregunta sobre la homogeneidad de estos escritos. Por otra, los distintos géneros literarios, tales como evangelio, epístola, apocalipsis y otros, también plantea interrogantes acerca de las semejanzas y diferencias literarias de estos textos.

Para abordar, entonces, las implicancias que ofrecen los estudios neotestamentarios a la teología sistemática, necesariamente hay que exponer algunos rasgos fundamentales de la exégesis en la actualidad. $\mathrm{Al}$ respecto, en esta presentación, dada la amplitud de estudios y perspectivas, habrá un énfasis en los evangelios, tanto en los sinópticos como en el de Juan. De esta forma, se comenzará con un esbozo de los caminos que ha tomado la exégesis en la historia de la Iglesia o, más bien, cómo los que han intentado comprender la Escritura lo han hecho en distintos momentos de la vida de la Iglesia, ya que los actuales paradigmas exegéticos se entienden a la luz de lo que se ha hecho en esta materia en el pasado. A continuación, se pondrá en evidencia cuáles han sido las características del método exegético que ha dominado la investigación en torno a los evangelios en el último siglo, para después pasar a exponer cuál es el paradigma exegético más difundido en la actualidad, para terminar con algunos desafíos que 
se presentan actualmente a la teología en relación con los estudios del Nuevo Testamento. Este breve recorrido permitirá detectar que el modo de aproximación a los evangelios, de parte de los investigadores, está en íntima relación con los modos de pensar y vivir de los seres humanos en distintos momentos de la historia y con algunas vicisitudes que se verificaron en esos momentos, y que estas diversas aproximaciones de una u otra manera se relacionan con el quehacer teológico.

\section{Paradigmas exegéticos ${ }^{1}$ en la historia de la Iglesia}

Es importante situar esta pregunta al interior de la comunidad eclesial. Tal vez hubiera bastado titular esta parte solamente "paradigmas exegéticos", pero el horizonte eclesial es sumamente relevante a la hora de preguntarse por qué se ha estudiado o se ha intentado comprender la Biblia, y en particular los evangelios, en los últimos dos mil años.

\subsection{Paradigma de los Padres de la Iglesia}

Los Padres de la Iglesia fueron verdaderos pioneros en la reflexión, debate, difusión y defensa de la fe en Jesucristo durante los primeros siglos cristianos, en los que muchas veces había una fuerte confusión. En ese contexto, el recurso a la Sagrada Escritura fue sumamente frecuente, ya que allí encontraban la fuente que animaba su reflexión para comprender y explicitar principalmente el misterio de Cristo.

Una vez que los textos neotestamentarios fueron considerados canónicos, proceso paulatino que requirió en algunos casos algo más de tiempo que en otros, los Padres se acercaron frecuentemente a dichos textos, generando así una rica reflexión. Sin pretender exponer con detalle la exégesis de los Padres, ni las particularidades que las diversas escuelas exegéticas, como la alejandrina y la antioqueña, presentaron en aquel entonces, el modo de acercarse a la comprensión de la Escritura tuvo ciertos elementos comunes.

1 En esta presentación se entiende por paradigma exegético a la perspectiva desde donde se intenta comprender el texto bíblico, lo cual implica un conjunto de precomprensiones acerca del texto y de las posibilidades que tiene el investigador para llegar a conocer el significado o el contenido del mismo. Se distingue del método exegético, ya que este es más bien el conjunto de procedimientos científicos de análisis literario para lograr una adecuada comprensión del texto. Un determinado paradigma exegético puede cristalizar en distintos métodos exegéticos. 
Para ellos, la Escritura era el lugar evidente desde donde argumentaron sus diversas posiciones. La exégesis era teología y la teología necesariamente era exegética. El pensar teológico muchas veces se planteó en términos polémicos, dada la proliferación de corrientes de pensamientos más o menos cristianos, paganos o judaizantes y, por consiguiente, el recurso a los textos sagrados se transformó en una fuente de inspiración para la necesaria argumentación en un ambiente marcado por la polémica.

Tres principios fueron más o menos una constante en ese periodo². En primer lugar, la comprensión de la Escritura debía tomar en cuenta que los textos tenían un sentido literal y también un sentido escondido. Evidentemente se tenía que partir tratando de explicitar el sentido literal que se percibía en el texto mismo, pero lo importante era llegar a su verdadero sentido, el que muchas veces permanece escondido en el texto. Para acceder a este sentido último, la alegoría o el simbolismo ofrece una gran ayuda, pues permite penetrar en el sentido escondido. Los Padres se dieron cuenta también que este paradigma, fundado solo en la alegoría, corría el riesgo de llegar a conclusiones divergentes, como de hecho ocurría, por ejemplo, en la polémica antignóstica ${ }^{3}$. Por este motivo, el primer principio debía ser iluminado por los otros dos, a saber, la regula fidei y la analogia fidei. La regula fidei correspondía a la confesión de fe declarada en el bautismo y transmitida por tradición. En tanto, la analogia fidei se basaba en el hecho de que la Biblia tiene un solo autor que es Dios, quien no se puede contradecir a sí mismo en el Antiguo y en el Nuevo Testamento; por consiguiente, hay una unidad coherente en toda la Escritura y en la verdades de la fe que encuentra su plenitud en la persona de Cristo, quien recapitula todo lo creado y lo increado.

Desde esta perspectiva, los Padres de la Iglesia, cada uno con su propia originalidad y con diversos matices, se transformaron en verdaderos comentaristas de la Biblia y esa era su teología.

2 Cf. P. Grech, «Ermeneutica», en P. Rossano - G. Ravasi - A. Girlanda (eds.) Nuovo Dizionario di Teologia Biblica (Cinisello Balsamo 1988), 466-474.

3 Así, por ejemplo, Ireneo en la confrontación contra los gnósticos percibió con claridad la necesidad de recurrir a un criterio objetivo, externo a la Biblia, para que se constituyera en referente que condujera al origen apostólico de la fe, cf. IRENEO DE Lyon, Adversus Haereses, I, 10,1. 


\subsection{Paradigma del Medievo ${ }^{4}$}

Durante la Edad Media hubo un fuerte acercamiento a la Escritura, dado el interés por tener una adecuada formación y, además, por la difusión de la lectio divina en los monasterios. En este contexto, se componen las catenae, verdaderas selecciones de textos patrísticos acerca diversos pasajes de la Biblia; célebre es, en este sentido, la catena aurea de Santo Tomás acerca de los cuatro evangelios.

El paradigma exegético se basaba en la distinción establecida por Agustín acerca de los cuatro sentidos de la Escritura: 1) literal, 2) etiológico, 3) analógico, y 4) alegórico 5 . A través del tiempo, se dio origen a la conocida formulación latina: Littera gesta docet; quid credas allegoria; moralis quid agas; quo tendas anagogia. Posteriormente, como una manera de simplificar la percepción acerca de los distintos sentidos del texto bíblico, Santo Tomás afirma que en realidad los sentidos son básicamente dos: litteralis y spiritualis; el primer significado de un término corresponde al sentido histórico o literal, mientras que el sentido espiritual, que ha de basarse en el literal, corresponde al significado de otros aspectos que se expresan a través del término ${ }^{6}$.

No obstante el énfasis de Santo Tomás en la importancia del sentido literal, la exégesis medieval tuvo una fuerte tendencia a la alegorización y esta tendencia dominó buena parte de los escritos acerca de la Biblia. Además, durante este periodo prácticamente se dio inicio al fenómeno de la separación entre exégesis y teología, de manera que ambas disciplinas comenzaron a seguir vías paralelas.

\subsection{Paradigmas de Lutero y de Trento}

La irrupción de Lutero en la historia trajo enormes consecuencias no solo para la vida de la Iglesia, sino también para la teología y la exégesis.

4 Cf. P. GreCH, «Ermeneutica», 474.

5 Cf. Agustín, De utilitate credendi, 3. En una línea similar, se sitúa Juan Casiano († hacia 435), quien también formula cuatro sentidos: 1) histórico o literal, 2) alegórico o cristológico, 3) tropológico, moral o antropológico, y 4) anagógico o escatológico, cf. R.E. Brown - S.M. Schneiders, «Hermenéutica», en R.E. Brown - J.A. Fitzmyer - R.E. Murphy (eds.), Nuevo Comentario Bíblico San Jerónimo, II (Estella 2004), 840.

6 Cf. Tomás de Aquino, Summa Theologiae, I, 1, 10. 
En oposición al modo escolástico de hacer teología y a la autoridad que se les concedía a los Padres de la Iglesia en la interpretación de los textos bíblicos, Lutero apela para que la sola Scriptura esté al centro de la Iglesia y de la experiencia cristiana, de manera que la Biblia sea intérprete de sí misma. Esta postura era coherente con su propuesta de justificación por la fe a través de Jesucristo. Su legado no se enmarcó tanto dentro de la exégesis cuanto al interior de la teología y de la actividad como traductor de la Biblia al alemán, pero desde el momento que pone la Palabra, y solo la Palabra, al centro de la experiencia religiosa, de hecho produjo en el ámbito protestante el abandono de la regula fidei como criterio de validación de la interpretación bíblica. Ya no es la Iglesia la que autentifica la Biblia, sino la Biblia la que autentifica la Iglesia, y, para que se verifique esta afirmación, necesariamente el sentido literal debe ser el único aceptado ${ }^{7}$. De esta forma, se rompe uno de los criterios hermenéuticos que había permanecido intocado en los anteriores paradigmas exegéticos.

La respuesta de la Iglesia católica no se dejó esperar. El Concilio de Trento, en la sesión IV del 8 de abril de 1546, no solo define dogmáticamente cuáles han de ser considerados libros canónicos y sagrados, sino también reafirma el principio interpretativo en cuanto que la comprensión del texto bíblico ha de hacerse en concordancia con la regula fidei; nadie puede, en materia de fe y costumbres, hacer una interpretación privada contra el sentido que mantiene la Iglesia y el consenso unánime de los Padres ${ }^{8}$. Este principio permanecerá con su valor normativo en los siglos siguientes y orientará el trabajo exegético en el ámbito católico, en tanto que la exégesis protestante seguirá su propio camino al margen de cualquier condicionamiento exógeno.

Entrando en el s. XVII un segundo problema se presenta en el horizonte cultural europeo que traerá consecuencias importantes para el estudio de la Biblia. Se trata de la nueva visión científica del mundo que encuentra en el caso Galileo Galilei su primer ejemplo dramático. Las observaciones de Galileo, siguiendo lo que Copérnico había señalado antes, pondrán sobre el tapete las preguntas acerca de la verdad de la Biblia y la verdad sobre el cosmos que propone el método científico. Galileo advirtió este problema y propuso distinguir entre las verdades

7 Cf. P. GreCH, «Ermeneutica», 474-475.

8 Cf. Enchiridion Biblicum, 57-60, 62. 
relativas a la fe y costumbres, en las que la Biblia no puede errar, de las verdades referidas al mundo natural, sobre las que la Sagrada Escritura transmite en lenguaje común aspectos que el conocimiento científico puede contradecir en un momento determinado. En consecuencia a lo determinado en el Concilio de Trento, la censura del Santo Oficio de febrero de 1616 declara herética la afirmación del heliocentrismo y de hecho no acepta la distinción propuesta por Galileo'. Este conflicto deja entrever lo que se vendrá después con el paradigma racionalista.

\subsection{Paradigma racionalista}

Los ss. XVII, XVIII y XIX fueron de gran actividad en torno a la Biblia en general y los evangelios en particular. Se considera que ha sido B. Spinoza (1632-1677) quien ha iniciado el movimiento racionalista; identifica a Dios con la naturaleza y el mundo, excluyendo que sea un Dios personal, sino más bien el impersonal orden geométrico que dirige el universo ${ }^{10}$. Erige la razón humana como el único criterio de comprensión válido y considera que cualquier realidad que escape al ámbito de lo racional no tiene posibilidad de existencia ${ }^{11}$.

Los postulados exegéticos de Spinoza causaron gran impacto. En el s. XVIII varios exégetas comienzan a estudiar la Escritura desde esta perspectiva, dando a luz propiamente el racionalismo en el ámbito exegético. Se trata de «la exclusión total o parcial de todo hecho o doctrina que no entra en los esquemas de la razón humana. Es el caso de los milagros, de las teofanías, de la encarnación, del nacimiento virginal y de la resurrección ${ }^{12}$. Evidentemente aquí se produce un punto de inflexión en los paradigmas exegéticos utilizados anteriormente en la Iglesia.

Los espacios abiertos y las conclusiones obtenidas por la puesta en práctica de esta nueva perspectiva no se dejaron esperar. En primer lugar, hubo un gran interés por reconstruir críticamente el texto bíblico. En el NT, J.J. Wettstein (1693-1754) da inicio a la moderna crítica textual con su edición crítica entre 1751-1754. Le siguen los trabajos

9 Cf. M. Pesce, «Dalla enciclica bíblica di Leone XIII Providentissimus Deus (1893) a quella di Pio XII Divino Afflante Spiritu (1943)» en AA. VV., Cento anni di cammino bíblico (Milano 1995) 41-44.

10 Cf. P. Grech, «Ermeneutica», 476-477.

11 Cf. B. SpInoza, Tractatus theologico-politicus, VII.

12 P. GRECH, «Ermeneutica», 477. 
de J.J. Griesbach (1745-1812), quien acuñó el término sinópticos para referirse a los tres primeros evangelios, K, Lachmann (1795-1851), C. von Tischendorf (1815-1874), B.F. Westcott (1825-1901) y J.A. Hort (1828-1892).

Sin embargo, en donde más da que hablar este nuevo paradigma es en el campo de la cristología. El primer aporte en esta línea es el de Reimarus, cuya obra fue publicada póstumamente; señala que Jesús no hizo milagros y que solo predicó el Reino de Dios; al final sus discípulos, a través de un astuto engaño, convencieron a sus contemporáneos que Jesús había resucitado, dando inicio así al cristianismo, cosa que no estaba en la mente de Jesús ${ }^{13}$. Después siguen varios otros intentos para explicar la vida de Jesús en clave racionalista, como la escuela de Tübingen, donde destacan D.F. Strauss, afirmando que cuanto se narra en los evangelios está impregnado de mito helénico ${ }^{14}$, y Baur quien pone en discusión, por ejemplo, el origen apostólico del cuarto evangelio, fechándolo hacia el año 170 , y por eso carente de todo valor histórico ${ }^{15}$.

De esta forma, el racionalismo dio origen a una gran cantidad de estudios bíblicos que fueron paulatinamente derribando muchas de las creencias sostenidas con respecto a los evangelios y los otros libros de la Escritura. No obstante los indiscutibles aportes que entregaron al conocimiento bíblico, entre los cuales el método exegético histórico-crítico, fue en definitiva un proceso de desacralización de la Sagrada Escritura. Por esta razón, los exégetas católicos seguían con interés y preocupación los estudios de sus colegas protestantes.

\subsection{Mutación del paradigma católico}

Desde fines del s. XIX el paradigma exegético del ámbito católico sufrió una interesante mutación, la que es posible advertir por medio de algunas intervenciones del magisterio pontificio. Sin pretender entregar una visión exhaustiva de este periodo, son básicamente tres las intervenciones en este tema, las que incidieron de manera significativa en el modo de hacer exégesis:

\footnotetext{
13 Cf. P. Grech, «Ermeneutica», 477.

14 Cf. D.F. Strauss, Das Leben Jesu, kritisch bearbeitet, I-II (Tübingen 1835), 1836.

15 Cf. F.C. Baur, Kritische Untersuchungen über die kanonischen Evangelien, ihr Verhältnis zueinander, ihr Charakter und Ursprung (Tübingen 1847).
} 
1) León XIII, encíclica Providentissimus Deus (1893): Publicada justo en el momento en que arreciaba el racionalismo de la exégesis liberal y, desde esa perspectiva, se criticaba fuertemente la interpretación católica de la Biblia. Contra lo que podría haber sucedido, la encíclica no propugnó un atrincheramiento frente al racionalismo, sino que más bien, apreciando el florecimiento de los estudios bíblicos de ese tipo, promueve vivamente el incentivo de la investigación en el campo bíblico. Sin embargo, continúa a pensar que el acceso a la Escritura es funcional a un sistema teológico, de corte neoescolástico, y, por eso, ha de ser usada para argumentar adecuadamente ${ }^{16}$. Finalmente, introduce una innovación con respecto a la exégesis que se estaba usando hasta ese momento entre los católicos al establecer algunos principios que han de regir la relación entre ciencia y teología en temáticas presentes en la Escritura, asumiendo en cierta forma cuanto había señalado Galileo en el s. XVI ${ }^{17}$. No obstante los buenos deseos de este documento, la polémica antimodernista que se produjo en las décadas siguientes, prácticamente paralizó la investigación histórica en el ámbito católico.

2) Pío XII, encíclica Divino Afflante Spiritu (1943): En un contexto totalmente distinto al anterior, esta encíclica da el paso decisivo para que la crítica histórica sea utilizada por los exégetas católicos. La motivación para tal toma de posición de parte del Papa se circunscribe en el cuestionamiento cada vez más fuerte que se estaba levantando contra no pocos estudiosos católicos que de hecho estaban utilizando este tipo de estudios. Era el llamado cuestionamiento espiritualista que veía con temor el avance de la exégesis histórico-crítica en la Iglesia ${ }^{18}$. La encíclica afirma

16 Llama la atención que la Providentissimus Deus plantea que la teología debe utilizar la Sagrada Escritura y que esta ha de ser casi el alma de la teología, cf. Enchiridion Biblicum, 114. Más tarde, en el Concilio Vaticano II, ya no se habla de utilización de la Sagrada Escritura, sino que la teología se apoya en la Escritura, porque contiene la palabra de Dios, y, por eso, debe ser el alma de la teología, cf. Concilio ecuménico Vaticano II, Constitución dogmática Dei Verbum sobre la divina revelación, 24.

17 Cf. Enchiridion Biblicum, 120-122 y M. Pesce, «Dalla enciclica bíblica di Leone XIII Providentissimus Deus (1893) a quella di Pio XII Divino Afflante Spiritu (1943)», 48-53.

18 De hecho, en 1941 la Pontificia Comisión Bíblica dirigió una carta al episcopado italiano poniendo en evidencia los injustos ataques que se expresan en un opúsculo anónimo de amplia difusión contra el estudio científico de la Sagrada Escritura. Este escrito constituye el primer documento de aceptación del método histórico 
que el punto de partida del estudio bíblico es el mismo texto; de aquí, entonces, le asigna un valor indiscutido a la crítica textual de los escritos originales $^{19}$. Enseguida, sostiene que la tarea suprema del exégeta es hallar y exponer el verdadero sentido de los textos de la Escritura, es decir, el sentido literal, de manera que aparezca claramente cuál es la mente o la intención del autor ${ }^{20}$; para mejor comprensión del sentido literal, la encíclica anima incluso a tomar en consideración los géneros literarios empleados por los hagiógrafos ${ }^{21}$. Asimismo exhorta a los exégetas a no olvidar el sentido espiritual de los textos sagrados, ya que su labor no se puede reducir solo a la exposición histórica, filológica o arqueológica de cuanto se narra en la Biblia ${ }^{22}$. Es difícil describir en pocas líneas el positivo impacto que produjo esta encíclica, especialmente en los círculos de estudiosos de la Biblia, ya que por primera vez se daba carta de ciudadanía católica a la aproximación histórico-crítica de la Escritura ${ }^{23}$.

3) Pontificia Comisión Bíblica, La interpretación de la Biblia en la Iglesia (1993): A 100 años de la Providentissimus Deus y a 50 de la Divino Afflante Spiritu, la Pontificia Comisión Bíblica publica un documento que también ha sido muy relevante con respecto a los paradigmas exegéticos aplicados por los estudiosos de la Biblia. El contexto nuevamente ha cambiado; ahora la amenaza proviene de la llamada lectura fundamentalista de la Escritura. Para el documento, «la lectura fundamentalista parte del principio de que, siendo la Biblia Palabra de Dios inspirada y exenta de error, debe ser leída e interpretada literalmente en todos sus detalles. Por "interpretación literal" entiende una interpretación primaria, literalista, es decir, que excluye todo esfuerzo de comprensión de la Biblia que tenga en cuenta su crecimiento histórico y su desarrollo. Se

crítico y será la base para que dos años después se escriba la Divino Afflante Spiritu por los 50 años de la Providentissimus Deus. Cf. Enchiridion Biblicum, 522-533.

19 Cf. Enchiridion Biblicum, 548.

20 Cf. Enchiridion Biblicum, 550-551.

21 Cf. Enchiridion Biblicum, 558-560.

22 Cf. Enchiridion Biblicum, 552-553.

23 Algunos años después hubo otras intervenciones pontificias que siguieron esta misma línea y llevaron a plenitud lo expuesto en la Divino Afflante Spiritu; es el caso de la Instrucción de la Pontificia Comisión Bíblica Sancta Mater Ecclesia sobre la verdad histórica de los Evangelios (1964), cf. Enchiridion Biblicum, 644-659, y la Constitución dogmática Dei Verbum sobre la divina revelación del Concilio Vaticano II (1965). 
opone, pues, al empleo del método histórico-crítico, así como de todo otro método científico para la interpretación de la Escritura. [...] El problema de base de esta lectura fundamentalista es que, rechazando tener en cuenta el carácter histórico de la revelación bíblica, se vuelve incapaz de aceptar plenamente la verdad de la Encarnación misma. [...] Rechaza admitir que la Palabra de Dios inspirada se ha expresado en lenguaje humano y que ha sido escrita, bajo la inspiración divina, por autores humanos, cuyas capacidades y posibilidades eran limitadas» ${ }^{24}$. La respuesta presentada en este documento a este tipo de lectura es en dos direcciones. Por una parte, reafirma el valor e importancia del método histórico-crítico en el estudio científico de la Sagrada Escritura, llegando a afirmar que es el método indispensable para entender el sentido de los textos antiguos ${ }^{25}$; incluso, insiste en el valor primordial del sentido literal de los textos, pero no olvida el sentido espiritual y afirma la posibilidad del sentido pleno (sensus plenior) ${ }^{26}$. Por otra, y he aquí su aporte más significativo para el tema que nos ocupa, presenta un interesante análisis de los nuevos métodos y acercamientos para el estudio bíblico, casi todos ellos dentro del paradigma exegético sincrónico. Llama positivamente la atención el esfuerzo del documento para poner en evidencia tanto los aportes significativos que cada método y acercamiento hace al estudio bíblico, así como también sus posibles limitaciones ${ }^{27}$. La única aproximación considerada totalmente inapropiada es justamente la lectura fundamentalista.

Teniendo en cuenta este panorama de los distintos paradigmas exegéticos en la historia de la Iglesia, que ciertamente debería ser complementado y matizado en muchos aspectos, se puede advertir un hilo conductor que no siempre ha tenido un solo componente, sino que a veces distintos de estos han seguido vías paralelas. Por prácticamente 15 siglos la exégesis buscó la comprensión de los textos bíblicos apoyándose

24 Enchiridion Biblicum, 1381 y 1384.

25 Al respecto señala: «El método histórico-crítico es el método indispensable para el estudio científico del sentido de los textos antiguos. Puesto que la Sagrada Escritura, en cuanto "Palabra de Dios en lenguaje humano", ha sido compuesta por autores humanos en todas sus partes y todas sus fuentes, su justa comprensión no solamente se admite como legítima, sino que requiere la utilización de este método", Enchiridion Biblicum, 1275.

26 Cf. Enchiridion Biblicum, 1402-1422.

27 Cf. Enchiridion Biblicum, 1291-1390. 
de manera más o menos explícita en la regula fidei y la analogia fidei. La irrupción de Lutero trajo como consecuencia, entre otras cosas, el abandono de la regula fidei, es decir, sacó al estudio bíblico de su matriz eclesial, con todo lo que esto podía implicar. Más adelante, el racionalismo produce una nueva escisión en el paradigma exegético, pues desvincula el estudio bíblico de la fe, dando inicio así a los estudios considerados científicos; en cierta forma, se pierde la analogia fidei. Paralelamente muchos exégetas seguían otras vías, tratando de sobrevivir dentro de los paradigmas anteriores, pero una teología de corte neoescolástico, orientada a usar la Escritura más que a estudiarla o a comprenderla, de una u otra forma y, tal vez sin pretenderlo, fue asfixiando las posibilidades de un auténtico estudio bíblico. La convergencia bíblica parecía que se iba a producir a mitad del $\mathrm{s}$. XX, pues el método histórico-crítico a partir de ese momento era universalmente aceptado y abría, entonces, enormes posibilidades de solución a las grandes interrogantes que se planteaban en el campo bíblico. Sin embargo, algunas décadas después un nuevo paradigma parece imponerse entre los estudiosos de la Biblia, que se expresa en los métodos sincrónicos de distinto signo. Una breve mirada al método histórico-crítico, especialmente aplicado a los evangelios, ayudará a captar qué ha ocurrido en estos últimos años.

\section{El MÉTOdO HISTÓRICO-CRÍTICO}

Este método se fue estructurando paulatinamente, a partir de las preguntas que se fueron planteando en el ámbito científico con respecto al texto bíblico. A riesgo de simplificarlo, sigue más o menos las siguientes etapas, especialmente en los evangelios:

a) Crítica textual: a partir del s. XVI comienza el interés, especialmente entre los protestantes, de hacer traducciones de la Biblia desde los textos originales. Surge así la edición griega del NT llamada textus receptus. Por varios siglos fue el texto de referencia, pero con la proliferación de investigaciones arqueológicas desde el s. XIX comenzaron a descubrirse una gran cantidad de papiros y códices del NT. Naturalmente el textus receptus, basado en pocos testigos, comienza a ser revisado, aparecen las ediciones críticas del NT y la crítica textual se afianza como disciplina científica que pretende determinar cuál eran los términos que componían el texto original. Sus aportes han sido muy significativos para clarificar muchas dudas textuales. Actualmente, en lo que respecta 
al NT, tenemos dos ediciones críticas que coinciden entre sí en el texto, pero son distintas en sus respectivos aparatos $\operatorname{críticos}^{28}$.

Si bien es cierto que la crítica textual ha resuelto muchas dudas, quedan todavía algunas por clarificar. Por ejemplo, Jn 20,31 dice: «Estos han sido escritos para que creáis que Jesús es el Cristo, el Hijo de Dios, y para que creyendo tengáis vida en su nombre». En castellano pareciera decir que el evangelio ha sido escrito para suscitar la fe en el lector. Sin embargo, viendo el texto griego de una edición crítica ese "para que creáis» es la traducción de íva $\pi\llcorner\sigma \tau \varepsilon v ́[\sigma] \eta \tau \varepsilon$, es decir, puede ser $\pi \iota \sigma \tau \varepsilon v ́ \sigma \eta \tau \varepsilon$ o $\pi \iota \sigma \tau \varepsilon u ́ \eta \tau \varepsilon$. El primero es aoristo subjuntivo y el segundo es presente subjuntivo. Ambas lecturas están adecuadamente atestiguadas. ¿Qué importancia tiene esto? Dependiendo de la lectura que se adopte, se podrá entender cuál es la finalidad del cuarto evangelio. Si se acepta la primera, se trataría de un aoristo ingresivo, es decir, señala el momento en el que se ingresa a un nuevo estado, en este caso la fe; por eso el evangelio de Juan tendría una finalidad misionera: suscitar la fe. En cambio, si se adopta la segunda versión, sintácticamente se estaría hablando de una situación que se está desarrollando en el tiempo, que no se inicia ni se acaba en ese momento; por consiguiente, el cuarto evangelio estaría destinado a incrementar y profundizar la fe y no a suscitarla; tendría una finalidad catequética. La discusión está abierta y la crítica textual no la ha podido resolver ${ }^{29}$.

b) Critica de las fuentes: Esta fue una de las primeras preocupaciones del análisis crítico. La simple comparación de los tres evangelios sinópticos dejaba entrever claramente que hay grandes semejanzas y no pocas diferencias entre ellos. La tarea de la crítica de las fuentes fue, entonces, dar una explicación plausible de esta constatación. Con no pocos detractores finalmente se impuso la hipótesis de las dos fuentes, que afirma la prioridad de Mc delante de los dos evangelios y una dependencia indirecta entre Mt y Lc que pasa por la denominada fuente

28 Cf. B. Aland - K. Aland - J. Karavidopoulos - C.M. Martini - B.M. MetzGer (eds.), The Greek New Testament (Stuttgart ${ }^{4} 1993$ ) y E. Nestle - K. Aland, ed., Novum Testamentum Graece. Apparatum criticum novis curis elaboraverunt Barbara et Kurt Aland una cum Instituto Studiorum textus Novi Testamenti Monasterii Westphaliae (Stuttgart ${ }^{27} 1993$ ).

29 Cf. B. M. Metzger, A Textual Commentary on the Greek New Testament (Stuttgart $\left.{ }^{2} 1994\right)$ 219-220. 
$\mathrm{Q}^{30}$. Esta hipótesis explica buena parte de las preguntas planteadas por la comparación de los sinópticos entre sí, pero abrió otras interrogantes significativas, como, por ejemplo, ¿por qué el evangelio de Mt sigue al de Mc, en circunstancias que Mateo habría sido un apóstol y Marcos no? En todo caso, con esta metodología comienza a ser evidente para los investigadores que los evangelios tienen una historia y que no fueron escritos de memoria por alguien en unas pocas horas de escritura. Detrás de cada evangelio hay fuentes, tal vez escritas, que están aportando abundante material para la elaboración de los textos.

Con respecto al cuarto evangelio, la crítica de las fuentes se ha visto menos exitosa, probablemente porque no tiene un texto similar con quien compararse, como es el caso de los sinópticos. Empero, varias hipótesis se han presentado para explicar el origen de Jn. Célebre es la propuesta de Bultmann, que marcó una tendencia imposible de evitar en casi toda la segunda mitad del s. $\mathrm{XX}^{31}$. Según este autor, el cuarto evangelio tuvo tres fuentes: la fuente de los signos, la fuente de los discursos de revelación y la fuente del relato de la pasión. La que gozó de mayor aceptación fue la fuente de los signos, semeia quelle; habría sido una fuente que narraba los 7 milagros descritos en el cuarto evangelio, pero presentarían una imagen de Jesús impregnada del mito griego del

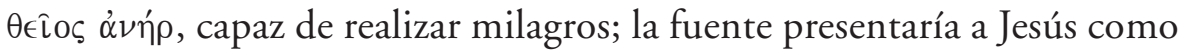
alguien capaz de hacer estos milagros, porque sería una prueba convincente para los destinatarios, quienes estarían impregnados de dicha concepción ${ }^{32}$. Con los aportes de varios exégetas, tales como Martyn,

30 Los inicios de esta hipótesis se encuentran en la exégesis alemana del s. XIX en autores tales como Lachmann, Weisse, Wilke. Holtzmann es quien enuncia los principios de esta hipótesis, cf. H.J. Holzmann, Die synoptischen Evangelien. Ihr Ursprung und geschichtlicher Charakter (Leipzig 1863). Sin embargo, son los exégetas ingleses quienes logran formularla en su versión depurada y más conocida, con una interesante variación propuesta por B.H. STreeter, The Four Gospels. A Study of Origins Treating of the Manuscript Tradition, Sources, Authorship \& Dates (London 1924).

31 Planteamiento presentado por este autor no de manera sistemática en su comentario al cuarto evangelio, cf. R. Bultmann, Das Evangelium des Johannes (Göttingen 1941).

32 Cf. W. Nicol, The Semeia in the Fourth Gospel: Tradition and Redaction (Leiden 1972), 48. Posteriormente, Van Belle, después de un exhaustivo análisis de todo cuanto se ha escrito acerca de esta hipótesis de Bultmann, concluye que no es una hipótesis necesaria para estudiar el cuarto evangelio, cf. G. van Belle, The Signs 
Brown y más recientemente Vidal, la atención cambió desde las eventuales fuentes del evangelio a la evolución que podría haber tenido el texto al interior de la comunidad joánica en consonancia a los distintos momentos históricos que tuvo que vivir esta comunidad en el transcurso del s. $\mathrm{I}^{33}$. El desplazamiento del interés hacia la actividad de las comunidades que han dado origen a los evangelios es lo que dio pie, entre otras cosas, a la siguiente etapa del método.

c) Crítica de las formas: Más conocida por su formulación en alemán: Formgeschichte ${ }^{34}$. Aquí es donde el método hace los aportes más sustanciales y controvertidos. Su punto de arranque es el intento de superar el punto de llegada de la crítica de las fuentes; ya no basta con saber qué texto es fuente de qué, sino más bien intentar descubrir o reconstruir la misma actividad de Jesús, qué hizo y qué dijo el Jesús histórico, a través del estudio del periodo prerredaccional de la comunidad pospascual. Estos estudios demostraron la importancia de las comunidades cristianas que transmitieron los recuerdos de Jesús a partir de sus necesidades vitales (Sitz im Leben) y que de una u otra forma los fueron modificando, por una parte, desde su convicción en la resurrección de Jesús y, por otra, desde las reglas habituales que se dan en la transmisión de una tradición dentro de una comunidad. Desde esta perspectiva, la crítica de las formas ve la necesidad de profundizar en los géneros literarios presentes en los evangelios, ya que son la forma habitual mediante la cual las comunidades fueron transmitiendo y modificando sus recuerdos de Jesús. El género literario permite percibir cuáles son los acentos existentes en el texto. Junto con eso, para el método es fundamental identificar los estratos redaccionales del texto, ya que de esta forma se puede acceder a la actividad literaria de la comunidad en distintos momentos prerredaccionales con el objeto de acercarse lo más posible al hecho Jesús.

Source in the Fourth Gospel. Historical Survey and Critical Evaluation of the Semeia Hypothesis (Leuven 1994), 376.

33 Cf. J.L. Martyn, History and Theology in the Fourth Gospel, Abingdon (Nashville ${ }^{2} 1979$ ); R.E. Brown, La comunidad del discipulo amado. Estudio de la eclesiología juánica (Salamanca 1983); S. VIDAL, Los escritos originales de la comunidad del discipulo "amigo" de Jesús (Salamanca 1997).

34 Los autores clásicos de la crítica de las formas son M. Dibelius, Die Formgeschichte des Evangeliums (Tübingen 1919) y R. Bultmann, Die Geschichte der synoptischen Tradition (Götingen 1921). 
La contribución de este tipo de análisis ha sido enorme; baste recordar, por ejemplo, la discusión en torno a la interpretación alegórica de las parábolas que inició Jülicher ${ }^{35}$ y que continuó Jeremias ${ }^{36}$. No obstante sus aportes, algunos presupuestos metodológicos de la crítica de las formas han suscitado una gran controversia. En primer lugar, el método parte de la base que los autores de los evangelios son meros compiladores, pues ellos ponen en el texto lo que han recibido de la tradición. En segundo lugar, la actividad de la comunidad es quien da origen a la tradición; su acción modela y modifica los recuerdos de tal forma que no es posible llegar al Jesús histórico; entre el texto y los acontecimientos históricos hay un velo puesto por la tradición que prácticamente es imposible traspasar. En tercer lugar, la excesiva preocupación por el momento tradicional condujo de hecho a una especie de abandono del texto en su forma final, tal como lo conocemos, de manera que se comenzó a prestar más atención en las hipotéticas reconstrucciones de los exégetas que en el texto mismo de los evangelios. Evidentemente estas perspectivas plantearon una serie de preguntas a los evangelios, especialmente en relación a su historicidad e inerrancia. Estas preguntas, junto al hecho de una cierta saturación por este tipo de análisis, permitió el advenimiento de la siguiente etapa.

d) Crítica de la redacción: también conocida como Redaktionsgeschichte. En la segunda mitad del s. XX comienza una revaloración de la actividad literaria de los autores de los evangelios, pues se advierte que ellos no fueron solo compiladores sino verdaderos teólogos que plasmaron su comprensión en la redacción de estos textos. En un primer momento, se siguió con la tendencia de distinguir los estratos redaccionales del texto, dejando atrás todo aquello que pudiera provenir de la tradición y centrando la atención en los marcos redaccionales que provendrían de la mano del autor de los evangelios. A poco andar se optó por considerar que en realidad todo el evangelio podría ser obra del autor, aunque haya recibido importante material reelaborado en el periodo tradicional. Si el autor escribe su obra, hace suyo cuanto ha recibido de otros. De esta forma, la crítica de la redacción pone la atención en descubrir la estructura del texto, la teología del evangelio y los condicionamientos

35 Cf. A. Jülicher, Die Gleichnisreden Jesu, I (Tübingen $\left.{ }^{2} 1899\right)$, 203-322.

36 Cf. J. Jeremias, Las parábolas de Jesús (Estella 1984), 29-142. 
socioculturales de la comunidad desde donde surge el texto y a quien en definitiva ha sido destinado ${ }^{37}$.

Los aportes de esta nueva aproximación a los evangelios han sido indiscutibles, ya que parecía que se volvía a reconstituir la unidad del texto, después de la avasalladora exégesis diseccionante de la crítica de las formas. Rápidamente se publicaron importantes obras en las que se presentaba el enfoque teológico de cada uno de los autores de los evangelios, poniendo de relieve la originalidad y genialidad de cada uno de ellos para entender especialmente el misterio en torno a la persona de Jesús ${ }^{38}$. Uno de los aspectos más trabajados por esta nueva perspectiva es el concepto de verdad de los textos de los evangelios. La crítica de las formas buscó llegar a las ipsissima verba Iesu, sin obtener resultados satisfactorios; en cambio, la crítica de la redacción enfatiza en el hecho que la verdad de los evangelios está en lo que testimonia la fe de los autores, quienes naturalmente han seleccionado el material empleado a partir justamente de sus convicciones como creyentes, los que pueden incluso llegar a ser no excesivamente prolijos en la transmisión de los datos de los hechos ${ }^{39}$.

Para algunos, una ulterior etapa del método histórico-crítico sería la historia de los efectos del texto, Wirkungsgeschichte. Esta perspectiva se basa en la siempre fecunda relación que hay entre un texto y sus lectores, más aún cuando los lectores se sitúan en distintas coordenadas de espacio y tiempo. Se trata de indagar cómo un pasaje o un libro de la Biblia ha influido en los distintos aspectos de la vida de una o más comunidades eclesiales ${ }^{40}$. No se puede negar que en algunas ocasiones se han verificado corrientes de interpretación tendenciosas que ha utilizado la Escritura para justificar acciones violentas tales como las discriminaciones raciales. Por este motivo, es necesario un adecuado discernimien-

37 Cf. E. Pérez-Cotapos, Introducción a los Evangelios sinópticos y Hechos de los Apóstoles, Apuntes de clase (Santiago 1995). 76-77.

38 Sobresalen en esta línea: W. MarXen, El evangelista Marcos. Estudio sobre la redacción del evangelio (Salamanca 1981); H. Conzelmann, El centro del tiempo. La teología de Lucas (Madrid 1974); W. Trilling, El verdadero Israel. Estudio de la teología de Mateo (Madrid 1974).

39 Cf. E. Pérez-Cotapos, Introducción a los Evangelios sinópticos y Hechos de los Apóstoles, 77.

40 Un muy interesante intento en esta línea es la obra J. GNILKa, El evangelio según San Marcos, I-II (Salamanca $\left.{ }^{2} 1992{ }^{2} 1993\right)$. 
to para evitar la apropiación indebida del texto por una determinada interpretación que se haya verificado en el tiempo tal como lo sugiere la Pontificia Comisión Bíblica en el documento antes señalado ${ }^{41}$.

\section{Nuevo paradigma, NueVos desafíos}

Con la crítica de la redacción, se volvió a considerar el texto de los evangelios como una unidad, o al menos como una realidad teológica unitaria. Esto permitió para que paulatinamente fueran apareciendo nuevas formas de interpretación del texto que tenían en común el considerarlo en su versión final, tal como se encuentra en la Escritura y tal como ha llegado a las comunidades cristianas hasta hoy. Contribuyó a esto una suerte de saturación del método histórico-crítico, pues cada vez más se extendía la impresión de que este método había dado todo o casi todo de cuanto estaba a su alcance. Había sido propuesto como el método científico de exégesis, que permitiría llegar a conclusiones aceptadas por todos; sin embargo, la verdad fue otra: una proliferación de propuestas basadas muchas veces en especulaciones difíciles de demostrar. Al nuevo paradigma, denominado sincrónico, no le interesa desenterrar los estratos de redacción del texto, ni profundizar en la historia que este ha recorrido para llegar a su versión final. Parte, más bien, de la convicción de que el texto del evangelio presenta una unidad acabada y que como tal constituye una unidad literaria con un contenido teológico coherente. Para comprender, entonces, su significado, se han de aplicar las técnicas del análisis literario.

Actualmente hay una verdadera explosión de tipos de análisis de los evangelios que está dando a luz una gran cantidad de literatura al respecto. Siguiendo el modo presentado en el documento La interpretación de la Biblia en la Iglesia, hay que distinguir entre método exegético y acercamiento a los texto bíblicos ${ }^{42}$.

Entre los métodos exegéticos, se destacan los que llevan a cabo un análisis literario del texto bíblico. Algunos de ellos están ofreciendo muy buenas perspectivas para comprender los evangelios como es el caso del

41 Cf. Enchiridion Biblicum, 1342.

42 "Por "método" exegético comprendemos un conjunto de procedimientos científicos puestos en acción para explicar las textos. Hablamos de "acercamiento" cuando se trata de una búsqueda orientada según un punto de vista particular», Enchiridion Biblicum, 1274, nota 1. 
análisis retórico que tiene tres acercamientos distintos: retórica clásica grecorromana, retórica semítica y la nueva retórica. En esta última se puede enmarcar el método llamado lingüístico-pragmático, que en un momento se pensó que podría dar muchas luces a las comunidades que viven el cristianismo en clave de compromiso en el mundo.

Otra vertiente que está dando muy buenos frutos es el análisis narrativo que toma en consideración las formas del relato y el testimonio, características de la comunicación humana.

El análisis semiótico o estructuralista se desarrolló hasta hace algunos años en ambientes de lengua francesa. Se crearon muchas expectativas en torno a los aportes que podría dar en el análisis bíblico, pero, al parecer, se ha ido diluyendo, tal vez por la complejidad de su método. Parecido a este pero mucho más simple es el método de estructura literaria, que pretende poner a la luz la estructura interna de los textos, es decir, la red de relaciones existentes entre los elementos que lo componen ${ }^{43}$.

Con respecto a los acercamientos, aquí se amplían aún más las posibilidades. El documento señalado presenta varios tipos de acercamiento. En primer lugar, los acercamientos basados en la tradición tales como el acercamiento canónico, el acercamiento desde las tradiciones judías de interpretación y la historia de los efectos del texto. También subraya el aporte de los acercamientos desde las ciencias humanas, como es el caso del acercamiento sociológico, el psicológico o psicoanalítico, que actualmente pareciera no estar rindiendo muchos frutos, y el acercamiento desde la antropología cultural. Este último ha hecho una rica contribución para entender algunos pasajes de los evangelios a partir de categorías como personalidad diádica, ordenamiento jerárquico del universo (especialmente sugerente para comprender los exorcismos), honor y deshonor, medio urbano y rural, etc. ${ }^{44}$.

43 Cf. J. CABA, «Métodos exegéticos en el estudio actual del Nuevo Testamento», Gregorianum 73 (1992), 643.

44 En este sentido son muy valiosos los aportes de B. Malina, El mundo del Nuevo Testamento. Perspectivas desde la antropología cultural (Estella 1995); B. Malina - R. Rohrbaugh, Los evangelios sinópticos y la cultura mediterránea del siglo I. Comentario desde las ciencias sociales (Estella 2002); B. Malina, El mundo social de Jesús y los evangelios (Santander 2002). 
Finalmente, nombra los acercamientos que llama contextuales o también denominados exégesis de propugnación ${ }^{45}$, entre los que se destacan el acercamiento liberacionista y el feminista.

Ante tal cantidad de métodos y acercamientos, se corre el riesgo de que los escritos sagrados se conviertan en fuente de divergencia entre los estudiosos. Por consiguiente, será necesario discernir adecuadamente en qué medida están aportando a clarificar y a comprender los textos o en qué medida están echando sobre ellos un velo que los esconde. Particularmente importante es esta pregunta en el caso de los acercamientos, ya que al no ser un método propiamente tal, se corre el riesgo de recurrir al texto desde horizontes totalmente distintos al bíblico y así extraer conclusiones ajenas al verdadero sentido literal del texto. El riesgo de la instrumentalización está siempre presente, especialmente cuando un acercamiento deviene en ideología.

\section{Desafíos para la teología sistemática}

El panorama histórico expuesto muestra la evolución que ha tenido la exégesis así como también qué relación ha tenido la teología con los estudios bíblicos. Si se pretende que el diálogo entre ambos sea fecundo, la Escritura ha de ser el alma de la teología, como lo señalara el Concilio ${ }^{46}$. Algunas pistas para abordar estos aspectos son las siguientes.

En primer lugar, la Palabra de Dios fue escrita como palabra humana por medio de personas concretas. La crítica de las formas nos ha ayudado a entender este proceso al interior de una comunidad, la que a su vez es una comunidad creyente. En este sentido, la fe se constituye en un elemento significativo para intentar comprender la labor de quienes escribieron esa Palabra de Dios. La fe condujo la Palabra de Dios desde la experiencia a la escritura. Por este motivo, la fe debe ayudar también a comunicar esa palabra desde la escritura a la teología y a la vida eclesial contemporánea. En otras palabras es lo que se ha denominado hermenéutica de la fe $e^{47}$.

45 Cf. R.E. Brown - S.M. SChneiders, «Hermenéutica», 850-851.

46 Cf. Concilio ecuménico Vaticano II, Constitución dogmática Dei Verbum sobre la divina revelación, 24 .

47 Cf. Benedicto XVI, Exhortación apostólica postsinodal Verbum Domini, 29. Aspecto ya tratado en un artículo publicado en 1989 , cuya traducción al castellano es J. Ratzinger, «La interpretación bíblica en conflicto. Sobre el problema de los 
Desde esta misma perspectiva, la teología fundada en la escritura ha de tomar en cuenta la tradición de la Iglesia, ya que esa tradición ha explicitado permanentemente el misterio de Dios que se revela en Jesucristo; lo ha vivido, celebrado y pensado.

Al momento de preguntarse por los métodos exegéticos, sobre todo en la actualidad en que se cuenta con abundantes métodos sincrónicos, y por los instrumentos filológicos, arqueológicos e históricos para comprender el texto bíblico, se ha de preguntar también por los presupuestos que los sustentan. Es evidente que todo instrumento de comprensión es bienvenido por el exégeta, pero a la hora de la interpretación hay que preguntarse por los presupuestos filosóficos de un determinado método para ver si efectivamente este puede ayudar a alcanzar el sentido de los textos. Un método que no deja espacio a la intervención o revelación de Dios en la historia, evidentemente no podrá colaborar de manera adecuada a una elaboración teológica ${ }^{48}$.

Por otra parte, para extraer la riqueza de la Palabra de Dios de manera que ilumine el quehacer teológico, hay que considerar a la Escritura en toda su complejidad y amplitud. Es la totalidad de la Biblia la que contiene esa Palabra y, por consiguiente, será la totalidad de ella misma la que ha de ofrecer esa Palabra. La segmentación del estudio bíblico ha de ser considerado un paso metodológico para después tener la comprensión unitaria de todas las partes.

Un aspecto delicado en la relación teología y exégesis, especialmente con respecto al Nuevo Testamento, se refiere a la cristología o, más bien, desde dónde se elabora la cristología. Es ya un lugar común la distancia que algunos estudiosos colocan entre el Jesús histórico y el Cristo de la fe. Los actuales métodos sincrónicos ofrecen una óptima oportunidad para no quedar entrampado en esa disyuntiva. $\mathrm{Al}$ respecto me parece pertinente citar a J. Ratzinger en su último libro, pues ofrece perspectivas que ayudan a superar esa tensión: «El "Jesús histórico", como aparece en la corriente principal de la exégesis crítica, basada en sus presupuestos hermenéuticos, es demasiado insignificante en su contenido como para ejercer una gran eficacia histórica; está excesivamente ambientado en el

fundamentos y la orientación de la exégesis hoy», en L. SÁNCHEz NaVArRo - C. Granados (eds.), Escritura e interpretación. Los fundamentos de la interpretación biblica (Madrid 2003), 19-54. 
pasado para dar buenas posibilidades de una relación personal con Él. [...] he tratado de desarrollar una mirada al Jesús de los Evangelios, un escucharle a Él que pudiera convertirse en un encuentro; pero también, en la escucha en comunión con los discípulos de Jesús de todos los tiempos, llegar a la certeza de la figura realmente histórica de Jesús» ${ }^{49}$.

Finalmente, una teología auténticamente bíblica será aquella que está atenta a dos polos. Por una parte, conoce bien la Escritura, usa los métodos exegéticos, se deja interpelar por la complejidad de la Biblia y, en definitiva, escucha la Palabra de Dios que está en ella. Y, por otra, conoce al hombre contemporáneo, sabe de sus búsquedas, atiende sus incertezas y escucha sus preguntas. Solo así la actividad pensante de la teología se transformará en un auténtico quehacer salvífico.

49 Cf. J. Ratzinger, Jesús de Nazaret. II. Desde la entrada en Jerusalén hasta la resurrección (Madrid 2011), 9. 
Resumen: El presente artículo pretende exponer el desarrollo que ha tenido la exégesis del Nuevo Testamento en la historia de los últimos dos milenios. Se constata, en primer lugar, que el aporte más significativo de la exégesis a la teología y a la vida de la Iglesia está en estrecha relación con la unión que el estudio bíblico tenga con la regula fidei. Asimismo, se explicita que el modo de aproximación a los evangelios, de parte de los investigadores, está en íntima relación con los modos de pensar y vivir de los seres humanos en distintos momentos de la historia y con algunas vicisitudes que se verificaron en esos momentos; estas diversas aproximaciones de una $\mathrm{u}$ otra manera se relacionan con el quehacer teológico.

Palabras clave: Nuevo Testamento, exégesis, hermenéutica, paradigmas exegéticos, métodos exegéticos, aproximaciones exegéticas, teología sistemática, método histórico-crítico.

Abstract: This article intends to present the development that New Testament exegesis has had in the history of the last two millennia. First, it confirms that the most significant contribution of exegesis to theology and to the life of the Church is closely related to the union between Bible study and the regula fidei. Also, it states that the way researchers approach the Gospels is intimately connected to the ways of thinking and living of human beings at different times in history and with some difficulties that were verified at the time; these various approaches relate in one way or another to the theological task.

Keywords: New Testament, exegesis, hermeneutics, exegetical paradigms, exegetical methods, exegetical approaches, systematic theology, historical critical method. 